\title{
TRA ANELITO D'INFINITO ED ESPERIENZA DEL REALE: PER UNA LETTURA DI ALCUNE POESIE DI ANTONIO GIAN GIURICIN
}

\author{
Sandro Cergna \\ Università Juraj Dobrila di Pola \\ Dipartimento di studi in lingua italiana
}

\section{Riassunto}

II presente contributo offre una lettura delle poesie in dialetto istrioto rovignese del poeta Antonio Gian Giuricin, pubblicate sulla rivista "La Battana" e in due Antologie delle opere premiate al Concorso d'Arte e di Cultura "Istria Nobilissima", edite negli anni 1986 e 1993. Nel lavoro se ne considerano alcuni aspetti tematici e stilistici rilevando come questi avvicinino i componimenti di Giuricin da un lato alla poetica dell'indefinito di ascendenza leopardiana, dall'altro alla poesia prosaica, naïf e bozzettistica, tipica dei topoi già sperimentati da Giusto Curto e Ligio Zanini, ma con sfumature, per certi accenti, originali rispetto ai due più noti interpreti della poesia in dialetto rovignese.

Parole chiave: poesia, istrioto, dialetto, Rovigno, Giuricin

I componimenti del rovignese Antonio Gian Giuric ${ }^{1}$ considerati in questo contributo, apparvero sulle pagine della "Battana" con il titolo Malincuneia, ${ }^{2}$ e in

\footnotetext{
${ }^{1}$ Antonio Gian Giuricin è nato a Rovigno nel 1923. Di professione economista, ha svolto per lunghi anni l'attività di responsabile bancario e funzionario amministrativo nella città natale. Nel biennio 1950-52 è stato direttore del giornale "Piassa Granda" e, dal 1955 al 1970 è stato corrispondente de "La Voce del Popolo" e di Radio Capodistria. Ha partecipato a numerosi concorsi di poesia riscuotendo notevole apprezzamento, di cui sono testimonianza premi e riconoscimenti. Tra gli incontri più importanti cui si è presentato con lavori di poesia, ricordiamo: "Istria Nobilissima" (1986, 1993), “Poesia in Piazza” (Muggia, 1984), “Antonio Bettanin" (Noventa Vicentina, 1990), "L' Ora dela prosa" (Pola, 1993). Nel 1994 ha pubblicato il libro di poesie "Chiaroscuri", mentre sue sillogi e poesie sparse si leggono nelle riviste "La Battana" (Fiume) e "MicRomania" (Bruxelles). È morto a Rovigno il 27 ottobre 1997.
} 
due numeri dell'Antologia delle opere premiate al Concorso d'Arte e di Cultura "Istria Nobilissima". ${ }^{3}$ Si tratta di una poesia di delicate ed effimere sensazioni che, pur rientrando, per molti versi, nel prolifico filone della tradizione rovignese, se ne discosta per una più pronunciata sensibilità nel ritrarre moti interiori, disposizioni d'animo ed emozioni, nel loro incerto e transitorio emergere alla coscienza. Ciò, naturalmente, non va inteso quale dominante della poesia giuriciniana. Anzi. È tuttavia indubbio che quando si eleva dal contingente prosastico e quotidiano che caratterizza, invece, Duman I (“Domani I"), Nenbadôure (“Cumuli di nembi”), in Malincuneia, e le poesie delle due Antologie, di cui si dirà avanti - il poeta rovignese riesce ad accarezzare il messaggio universale della poesia. Così, attraverso gli instabili indizi del ricordo, ormai / inpacadi in Laghi nuiufi / - / racchiusi in svaghi noiosi / (Incaleîa - "Indolenza"), o quelli di un'appena sfiorata esperienza, quando l'animo è / inbutilgià d'insierto / - / imbottigliato d'incerto / (Atifa - "Attesa"), o ancora attraverso le non meno effimere voci e suoni che ritornano alla mente come un'eco / al raciamo viseîn da bufe in festa / - / al richiamo vicino di voci festanti / (Malincuneia - "Malinconia"), Giuricin si avvicina ad esprimere l'ineluttabile fugacità del tempo; ad interloquire con il sé più recondito in /oûn abandon scunfinà " - / uno sconfinato abbandono / (Indifarensa _ "Indifferenza"); ad esprimere mirabili figure, passaggi e motivi sinestetici, che si possono ritrovare in molte delle poesie qui prese in esame.

C'è, quindi, in Giuricin una disposizione all'effimero, un'affinità con il transeunte di cui si fa lodevole cantore, con immersioni nei moti sommessi e intimi dell'animo, negli aggrovigliamenti interiori più riposti. Il suo è uno scandagliare, con tocco leggero, le profondità del proprio io. È il caso, per esempio, di Indifarensa (Indifferenza) in cui l'autore registra, quasi con un sofisticatissimo strumento, / Indeîsi Jgradeidi / [che] i fa da isca cun prufonda incaleîa, / feîn da altarà oûn gradeîto da/misià / - / Sgradevoli presagi / [che] stanno adescando con profonda indolenza, / fino a turbare un piacevole risveglio /. ${ }^{4}$

\footnotetext{
${ }^{2}$ L'articolo, cui si accompagnano otto poesie del poeta rovignese, è di Elis Deghenghi Olujić, Antonio Gian Giuricin, pubblicato in "La Battana" A. XXXV n. 127, Fiume, Edit, 1998, pp. 35-45.

${ }^{3}$ Rispettivamente, l'Antologia del 1986 riporta una plaquette di quattro poesie, El cor del mondo (II cuore del mondo) con la quale l'autore ottenne la menzione onorevole per la poesia; nell'Antologia del 1993 è pubblicata una raccolta di nove poesie dal titolo $D^{\prime}$ in trasto in sinteîna (Di palo in frasca), con le quali l'autore vinse il Secondo premio per la poesia dialettale.

4 “La Battana”, cit., p. 43.
} 
Questo sottile equilibrismo psicologico, al quale l'autore si accosta con il già rilevato / anamo inbutilgià d'insierto / - / animo imbottigliato d'incerto $/{ }^{5}$ si fa particolarmente intuire nella poesia Nutoûrno (Notturno), in cui momentanee sensazioni liminali escono in vivide percezioni sinestetiche che proiettano il lettore in un mondo irreale e fantastico, tra la / quitisa / - / quiete / della realtà esterna e il canto della propria intimità, e dove si possono / vidi imagine sfumade ca fulasa / cume ca fuoso murbade pioûme al vento / - / vedere immagini evanescenti svolazzare / come fossero morbide piume al vento /. ${ }^{6}$ Giuricin, così, in maniera originale, anche se solo a brevi riprese, si distacca dalla pura lirica marina e marinaresca, e da quella dell'ambiente naturale e rivierasco, tipici della poesia dialettale rovignese, proiettando invece la sua sensibilità verso vibrazioni nuove e inusuali, poco presenti fino ad allora nell'affermata tradizione poetica istriota rovignese. In questo, senza dubbio, possiamo cogliere una nota di originalità del poeta rispetto ai contemporanei Zanini e Curto, ma anche, in seguito, rispetto a quelli che saranno i tratti più salienti della poesia dei fratelli Libero e Vlado Benussi. Ritornando ancora un momento a Nutoûrno, è altresì da notare come il componimento costituisca, con la sua forte carica onirico-fantastica e col suo toccare le corde dell'intima sensibilità del poeta, un esempio di rinnovamento nella stagione neodialettale istriana, a dimostrazione della duttilità e delle potenzialità espressive del codice vernacolo istrioto. La poesia, ancora, mi sembra rappresenti il conseguimento, per l'autore, di una condizione di equilibrio tra la propria interiorità, che è / spasio preîvo anca da murmurii / - / spazio privo anche di mormorii /, e la concretezza esterna, / lifer buligoûn da la nuoto / - / leggero brusio notturno /; in tal senso può esser letta come la rivelazione di uno stato di serenità, raggiunto attraverso un effimero gioco speculare tra i propri moti psicologici e l'esperienza immanente della realtà. Se poi ci si sofferma su alcune immagini chiave della poesia (leggero brusio notturno, solo quiete, voci sommesse, immagini evanescenti, successioni senza fine), è evidente nel rovignese l'influsso dell'idillio leopardiano L'Infinito. Anche in Giuricin, infatti, è dalla discrepanza della sovrapposizione dei due piani, quello dell'interiorità e quello della realtà esterna, che può rivelarsi, / in oûn mumento / - / in un istante / il noumeno: ciò che per Leopardi sta oltre la siepe, e che in Giuricin sono / imagine sfumade / - / immagini evanescenti /, / murbade pioûme al vento / - / morbide piume al vento /; in entrambi i casi pura immaginazione, ritorno al primigenio, all'originario, all'antico. $\mathrm{E}$, anche qui, anelito accorato, poiché irrealizzabile, alla trascendenza, riconoscibile nell'ultimo verso, che è chiara invocazione dell'infinito: / ca li sa ripietiso sensa mai

\footnotetext{
${ }^{5}$ Dalla poesia Atifa (Attesa), ivi, p. 42.

${ }^{6}$ Ivi, p. 40.
} 
farmase / - / che si ripetessero in successione senza fine /. In questo senso, anche i versi di Giuricin non attingono a quella che Leopardi definisce "poesia del sentimento", cioè dei moderni, ma, per dirla col Gioanola, "del puro sentire, Befindlichkeit nei termini dell'esistenzialismo heideggeriano, l'accorgersi cioè della propria condizione infelice, consegnata alla morte, e della propria inappartenenza all'essere". ${ }^{7}$ Quanta eco, infatti, tra quello / spasio preîvo anca da murmurii / - / spazio privo anche di mormorii / e quegli "interminati / spazi [...], e sovrumani / silenzi, e profondissima quiete", cui risponde subito appresso quel / inturno e pioûn in là, / sulu ca quitisa / - / attorno e discosto, / solo quiete /. Ancora: così come ne L'Infinito a esplicitare l'opposizione "su cui tutta la composizione si struttura, tra il qui e ora dell'esistenza (ridotta alla tracce rappresentate dal colle, dalla siepe, dalle piante e dal vento) e l'esperienza dell'altrove, come denunciano morfologicamente le due serie degli aggettivi dimostrativi contrapposti 'questo' e 'quello'"8 troviamo il gerundio "comparando", così in Nutoûrno la medesima funzione chiave è svolta dall'avverbio / In quil / - / Nel mentre / posto, inoltre, in posizione privilegiata, a metà componimento, vero e proprio spartiacque, anche qui, tra il momento dell'immanenza $V$ oûn lifer buligoûn da la nuoto / - / un leggero brusio notturno $/$ e quello dell'esperienza dell'altrove, riecheggiato nella seconda parte della poesia.

\section{Nutoûrno}

In oûn lifer buligoûn da la nuoto

ca sa lu vularavo sensa umiliante inseîdie,

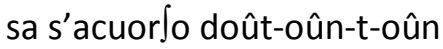

oûn spasio preîvo anca da murmurii

e inturno e pioûn in là,

sulu ca quitisa.

In quil,

drento da si stisi in oûn mumento,

a par da scultà bufe $\int$ mursade in carsento

ca pian pianeîn li sa trasfurma in canto

e vidi imagine sfumade ca Jvulasa

cume ca fuoso murbade pioûme al vento,

ca rifleise dai ragi de la loûna Jura da el mar,

sa li vularavo,

\footnotetext{
${ }^{7}$ GIOANOLA, E., 2005, Psicanalisi e interpretazione letteraria, Milano, Jaca Book, p. 73.

${ }^{8}$ Ivi, p. 85.
} 
insieme a quil canto,

ca li sa ripietiso sensa mai farmase. ${ }^{9}$

Il motivo dell'auscultazione interiore è presente pure in altri degli otto componimenti proposti nelle pagine de "La Battana". Indicativi sono, a questo proposito, già i titoli di essi: Incaleîa (Indolenza), Malincuneia (Malinconia), Difiderio (Desiderio), accanto ai già citati Atifa e Indifarensa. In tutte le composizioni vi è un che di aleatorio, di sfuggente, dato dal ricorso dell'autore a espressioni e parole chiave quali: sogni svogliati, ricordi consunti, svaghi noiosi (Incaleîa); sguardo placato, sprovveduto oblio (Malincuneia); sogni (Difiderio), che donano ai versi un tono quasi attutito da un'intima, pacata meditazione del poeta con se stesso e con il proprio vissuto. I sei componimenti, infatti, sono legati da un unico, comune filo conduttore: la rappresentazione, attraverso i versi, di uno stato di abbandono e di resa, quasi di catatonico ripiegamento in se stesso; un immobilismo che si rivela già, come visto sopra, dagli stessi titoli. In Incaleîa, così, il poeta descrive un'esistenza priva di alcun contenuto: immerso in un vuoto e in un'assenza assoluti, l'io poetico si fa qui testimone di una vita / sensa stuorie, / sensa scuosi, / sensa rumuri/ - /senza storie, / senza scosse, / senza rumori /, fatta di giorni che / scuro tratignoûdi / - /scorrono rallentati /, e dove I'unico accadimento è il ripetersi incessante di tale coscienza del nulla. A trasparire è anche qui, chiaramente resa dalla sequenza delle negazioni avverbiali "senza" e dal quasi ossimoro "scorrono rallentati", una sensazione di assenza di attività, di esclusione dalla realtà e di ritiro dell'io poetico in se stesso. È ciò che Gioanola definisce "rifiuto del movimento, tipico della malinconia, trattandosi appunto di una condizione di morte-in-vita, che abolisce i tratti caratteristici della vitalità". ${ }^{10}$

Il quadro di un'immobilità assoluta emerge pure da Malincuneia, dove lo sguardo dell'io poetico è colto nella sua rigida fissità, / inichideî / - / inerte /, in opposizione, invece, al movimento scompigliato delle nubi, che come un vortice attrae e risucchia quello sguardo in sé, rendendolo insensibile, infine, anche / al raciamo viseîn da buje in festa / - / al richiamo vicino di voci festanti /. Mi sembra molto efficace, oltre che bella, l'immagine che qui Giuricin adotta per delineare,

\footnotetext{
${ }^{9}$ Ivi, p. 40. "Notturno. In un leggero brusio notturno / che lo si invoca senza insidie umilianti, / s'avverte d'un tratto / uno spazio privo anche di mormorii / e attorno e discosto, / solo quiete. / Nel mentre, / dentro di sé in un istante, / sembra ascoltare un crescendo di voci sommesse / che si trasformano pian piano in canto / e vedere immagini evanescenti svolazzare / come fossero morbide piume al vento, / che riflesse dai raggi lunari sul mare, / si vorrebbe, / assieme a quel canto, / che si ripetessero in successioni senza fine".

${ }^{10}$ GioAnola, E., cit., p. 83.
} 
anche icasticamente, il contrasto tra apatia e inerzia da un lato, e moto, vitalità dall'altro. Immagine, questa del contrasto, che ritorna anche, seppure resa con più leggeri ed effimeri tratteggi, in Atifa, Difiderio e Indifarensa.

Ma accanto al motivo intimistico, Giuricin non tralascia di rappresentare nei suoi versi la natura e l'ambiente rivierasco rovignese, con i suoi luoghi caratteristici, le sue calli e contrade, autentico e imprescindibile topos della "scuola" rovignese. Dalla voce terrigna, più grave e agreste di Curto, però, o da quella più intellettualistica, ma sempre radicata nel locus litoraneo rovignese di Zanini, Giuricin si discosta - come pure dagli accenti più tormentati dei Benussi per una fedele, quanto non raramente ingenua trasposizione delle bellezze naturali della città in vivaci quadretti, caratterizzati da un impressionismo colorito e immediato, spesso prosastico e bonario. È il caso, per esempio, delle poesie che compongono le sillogi El cor del mondo (II cuore del mondo) ${ }^{11}$ e $D^{\prime}$ in trasto in sinteîna (Di palo in frasca). ${ }^{12}$ Nella prima, ad esempio, la sua cuntrada è resa con squarci di incisiva e rapida icasticità, quasi come le sequenze di un cortometraggio:

Fimane santade ca ciaculiva su i scalideîni

o dinanti a i purtoni,

Jento ca pasiva indafarada,

nui fioi ca sa Jughiva,

muriede ca cantuliva dastrigando la cala

e gati per i cantoni ${ }^{13}$

avvicinandosi, per questo aspetto, alla pennellata svelta e concisa di Virgilio Giotti nella raffigurazione della Trieste del suo tempo o della stessa Rovigno. ${ }^{14}$ Ritornando a La mieîa cuntrada, il poeta rovignese appena nel penultimo verso ci fa capire che quello che ha dipinto con vivaci guizzi di parole è il quadro che gli "ricorda la [sua] contrada", aggiungendo, in chiusa, con tocco da maestro, l'ultimo schizzo: / cu i drapi culuradi ca sa sughiva par i barconi / - / con i panni colorati che si asciugavano sulle finestre /.

\footnotetext{
${ }^{11}$ In Antologia "Istria Nobilissima", Fiume-Trieste, UIIF-UPT, 1986, pp. 99-107.

${ }^{12}$ In Antologia "Istria Nobilissima”, Fiume-Trieste, UIIF-UPT, 1993, pp. 37-46.

${ }^{13}$ Dalla poesia La meîa cuntrada (La mia contrada), in Antologia "Istria Nobilissima", 1986, cit., p. 102. "Donne sedute che chiacchieravano sui gradini / o davanti ai portoni, / gente che passava indaffarata, / noi bambini che giocavamo, / ragazze che canticchiavano affaccendate in casa / e gatti per gli angoli".

${ }^{14}$ ROVIGNO. lero a Rovigno, rivado / quela matina bonora: / me iero sentado fora / su 'na carega in $t$ un bar. // lero là in Piazza de le erbe, / batuda de contadini, / de piazzarioi, de fachini, / de cari e mussi. [...] lero a Rovigno, là in Istria! / Rovigno, con quel su' grosso / campanilon che vien dosso, / piantado bianco lassù [...]. In CecovinI M., Fraulini M., Honoré Bianchi O., Maier B., Todeschini F. (a cura di), 1991, Scrittori triestini del Novecento. Antologia, Trieste, Lint ${ }^{2}$, pp. 636-637.
} 
Ma caldi e suggestivi affreschi, che ritraggono una natura quasi incantata, sono quelli, ancora, affidati a brevi componimenti, quali El cor del mondo, Cu 'I sul a Ruveîgno a sa Jbasa (Rovigno al tramonto) o la bellissima Panurama sul mar (Panorama sul mare) dove, in pochi ed essenziali versi, il poeta riesce a condensare un'immagine densa di fluidi colori che sfociano, nell'ultimo verso, in un fervido e appassionato appello alla vita.

Teînte turcheîne a miera, panalade a piena man

scunfondo là, fora el scuio da Bagnole,

sil e mar

int'oûn simineîgo farmantà da splandoûri

ca fa ravidi da iefi veîvi. ${ }^{15}$

Colorati e pervasi di scoppiettante vitalità sono pure i componimenti più lunghi, quasi brevi storielle, in cui Giuricin indugia sull'elenco toponomastico (Ruveîgno bielo - Rovigno bella), o sul rilievo del particolare impreziosito da vivaci e fulgidi quadretti naïf. Ecco come, con una mirabile similitudine, l'autore ci presenta la vista di Rovigno dall'alto del Monte:

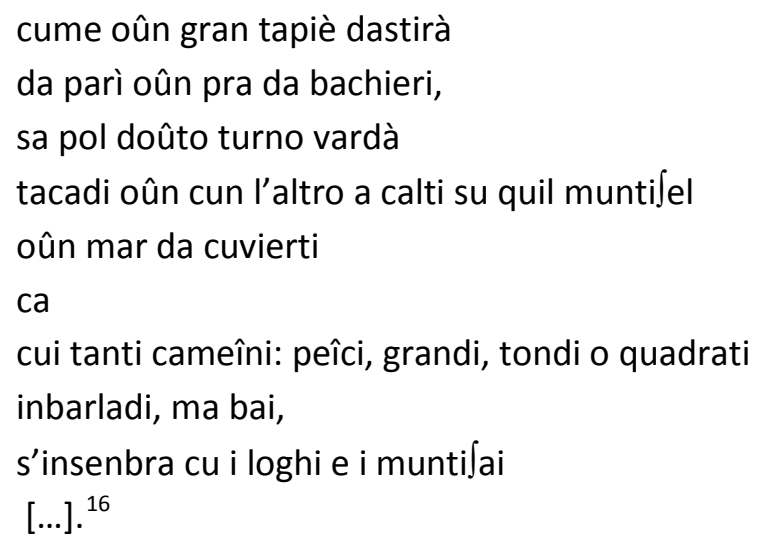

\footnotetext{
${ }^{15}$ Antologia "Istria Nobilissima”, 1986, cit., p. 104. "Miriadi di variazioni d'azzurro / pennellate a distesa / confondono, di là dallo scoglio di Bagnole, / cielo e mare / in un'abbagliante fantasmagorica luce / che ci fa richiamare d'esser vivi".

${ }^{16}$ Da El campaneîl da Sant'Ufiemia, in Antologia "Istria Nobilissima", 1993, cit., p. 40. "come un gran tappeto steso / da sembrare un prato di papaveri, / si possono tutt'attorno osservare, / attaccati uno con l'altro a ripiani su quella collinetta / un mare di tetti / che / con i tanti comignoli: piccoli, grandi, rotondi o quadrati, / sbilenchi ma eleganti, / si uniformano con i campi e le collinette / [...].
} 
Brevi narrazioni in versi sono pure le poesie Cumpagnamento da oûn viecio pascadour (Funerale di un vecchio pescatore) e L'oûltimo samarol (L'ultimo somarello); nella prima, il poeta con voce sommessa rappresenta il triste corteo dei rovignesi che rendono le esequie accompagnando, / cume in oûn ciapo da pisi da paso / ca in schera va par la curanteîa veîa / - / come un banco di pesci di passaggio / che, incolonnati, seguono la corrente /, il defunto alla sepoltura. Nella seconda poesia, la scomparsa dell'ultimo somarello è occasione per il poeta di un'accorata rievocazione di un tempo irrimediabilmente trascorso; un tempo quasi mitico, di cui Giuricin mette in risalto lo stretto rapporto che accomunava l'uomo all'animale e alla terra, qui tratteggiato con fluenti immagini in cui si esprimono, con plastica intensità, paesaggi e umori di un mondo ormai scomparso:

\section{[...] \\ E cul paron pusiva su la iera rento el tagoûr magnando oûn bucon, oûn tuoco da pan el ga butiva parsioû ca el sa fago anca loû la buca bona. ${ }^{17}$}

Concludendo, si può dire che attraverso la poesia, Giuricin riesce a esprimere, da un lato, il proprio mondo interiore, fatto di baluginii e di ripescamenti dall'inconscio, tratteggiati con accenti a volte malinconici che gli derivano, come si è cercato di mostrare, da una non superficiale frequentazione dei classici della poesia italiana, e di Leopardi in particolare; a tale proposito, possiamo qui accennare al pensiero del Lavagetto, secondo il quale "L'atto poetico, che può giovarsi di tutte le risorse dell'inconscio e che dall'inconscio attinge continuamente, è anche e soprattutto la più decisa affermazione della coscienza nel momento in cui trasforma quella materia in un prodotto finito". ${ }^{18}$ Dall'altro lato, il poeta rovignese attraverso i suoi versi attinge al concreto e all'immediato del piccolo mondo di Rovigno, fatto di atti e di atteggiamenti semplici, spesso umili, ma profondamente radicati nell'esperienza e nell'immaginario giuriciniano e che, quando emergono, spiccano per accenti di genuina e, a tratti, limpida, cristallina poesia.

\footnotetext{
${ }^{17}$ Ivi, p. 45. " [...] E mentre il padrone riposava sull'aia vicino al tugurio / mangiando un boccone, / un pezzo di pane gli gettava / affinché assaggiasse anche lui il buon sapore".

${ }^{18}$ LaVAgetto M., La gallina di Saba, citato in E. Gioanola, cit., p. 45.
} 


\section{BIBLIOGRAFIA}

CECOVINI, M., FRAULINI, M., HONORÉ BIANCHI, O., MAIER, B., TODESCHINI, F. (a cura di), $1991^{2}$, Scrittori triestini del Novecento. Antologia, Lint, Trieste.

DEGHENGHI OLUJIĆ, E., 1998, Antonio Gian Giuricin, in "La Battana” A. XXXV n. 127, Edit, Fiume-Rijeka.

GIOANOLA, E., 2005, Psicanalisi e interpretazione letteraria, Jaca Book, Torino.

GIURICIN, A. G., 1986, El cor del mondo, in Antologia delle opere premiate al Concorso d'Arte e di Cultura "Istria Nobilissima", UPT-UIIF, Fiume-Rijeka.

GIURICIN, A. G., 1993, D'in trasto in sinteîna, in Antologia delle opere premiate al Concorso d'Arte e di Cultura "Istria Nobilissima", UPT-UIIF, Fiume-Rijeka.

LAVAGETTO M., La gallina di Saba, in GIOANOLA E., 2005, Psicanalisi e interpretazione letteraria, Jaca Book, Torino.

\section{IZMEĐU ŽUDNJE ZA BESKONAČNIM I ISKUSTVA STVARNOSTI: INTERPRETACIJA POJEDINIH PJESAMA ANTONIJA GIANA GIURICINA}

Ovaj članak prikazuje interpretaciju pjesama na rovinjskom istriotskom dijalektu pjesnika Antonija Giana Giuricina, objavljenih u časopisu „La Battana“ i u dvjema Antologijama nagrađenih djela na natječaju za umjetnost i kulturu „Istria Nobilissima“, izdanih 1986. i 1993. godine. U radu se razmatraju pojedini tematski i stilistički aspekti koji s jedne strane otkrivaju kako se Giuricinova djela približavaju poeziji neodređenosti svojstvenoj Leopardiju, a s druge strane prozaičnoj, naivnoj i manirističkoj poeziji tipičnoj za topose kojima su se već bavili Giusto Curto i Ligio Zanini, no s karakterističnim prinosima u odnosu na dvojicu poznatijih rovinjskih pjesnika.

Ključne riječi: poezija, istriotski/istroromanski, dijalekt, Rovinj, Giuricin

\section{BETWEEN LONGING FOR INFINITY AND EXPERIENCING REALITY: AN INTERPRETATION OF INDIVIDUAL POEMS OF ANTONIO GIAN GIURICIN}

This article presents an interpretation of the poems in the Rovinj Istriot dialect by the poet Antonio Gian Giuricin, published in the "La Battana" journal and in two Anthologies, awarded in the "Istria Nobilissima" arts and culture contest, published in 1986 and 1993. This paper examines certain thematic and stylistic aspects discovering, on the one hand, how Giuricin's work is getting closer to the poetry of infinity inherent to Leopardi, and on the other, to the prosaic, naive and manneristic poetry typical of the topoi that Giusto Curto and Ligio Zanini had already dealt with, however, with, in some respects, original expressions in relation to the two better known poets in the Rovinj dialect.

Keywords: poetry, Istriot/Istroromance, dialect, Rovinj, Giuricin 